\title{
FERRAMENTAS PARA MODELAÇÃO AUTOMÁTICA SCAN-TO-BIM
}

\author{
Luís Sanhudo $^{(1)}$, João Poças Martins ${ }^{(1)}$, Nuno Ramos ${ }^{(2)}$ \\ (1) CONSTRUCT-GEQUALTEC, Faculdade de Engenharia da Universidade do Porto, Porto \\ (2) CONSTRUCT-LFC, Faculdade de Engenharia da Universidade do Porto, Porto
}

\begin{abstract}
Resumo
Nos últimos anos, apesar da crescente necessidade de obter de forma precisa e automática modelos BIM semanticamente enriquecidos de edifícios existentes, atualmente, nenhum software permite a passagem direta de nuvens de pontos para BIM. De facto, apesar de recente software permitir a importação de nuvens de pontos para ambientes BIM, o processo de modelação permanece amplamente manual, demorado, subjetivo e propenso a erros.

Com o intuito de auxiliar a resolução deste problema, os autores propuseram anteriormente uma metodologia na qual algoritmos de aprendizagem computacional possibilitam a segmentação e identificação de elementos construtivos numa nuvem de pontos, seguida da sua posterior modelação automática em BIM [1]. Contudo, para possibilitar o treino destes algoritmos, é necessário realizar um levantamento de uma substancial quantidade de nuvens de pontos. Com o objetivo de contornar este obstáculo, o presente artigo apresenta duas ferramentas digitais que interagem num ambiente virtual BIM, gerando nuvens de pontos artificiais de modelos previamente criados. A primeira ferramenta tem como objetivo o posicionamento otimizado de estações laser scanner no ambiente BIM, enquanto a segunda simula o funcionamento do laser scanner. Em conjunto, estas ferramentas permitem a criação expedita de um número elevado de nuvens de pontos já categorizadas, que serão depois utilizadas para a criação de uma base de dados para treinar o software de aprendizagem computacional.
\end{abstract}

\section{Introdução}

O volume de projetos de alteração, ampliação, manutenção, reparação, renovação, remodelação e reabilitação tem vindo a crescer com a gradual expansão do edificado existente [2]. Com o objetivo de auxiliar a execução destes projetos, novas tecnologias para levantamento de geometria as-is do edifício têm sido exploradas no sector da Construção. Nomeadamente, o laser scanning oferece uma forma expedita e precisa de obter a geometria de um edifício [3-5]. Contudo, apesar de recentes esforços para a importação de nuvens de pontos para ambientes BIM, o subsequente processo de modelação permanece ainda amplamente manual, demorado, trabalhoso, subjetivo e propenso a erros [6,7]. Neste sentido, múltiplos trabalhos [6-11] referem uma crescente necessidade de desenvolver um método para a obtenção automática e precisa de modelos 3D semanticamente enriquecidos, eliminando uma porção significativa do processo 
de modelação através da passagem direta de nuvens de pontos para modelos BIM - solução denominada de "Scan-to-BIM". Para desenvolver esta solução, a literatura atual identifica três passos a serem automatizados: segmentação da nuvem de pontos; classificação dos segmentos obtidos em elementos construtivos; e modelação destes elementos em ambiente BIM.

Tendo já proposto uma metodologia para desenvolvimento desta solução, bem como resolvido o primeiro passo [1], no presente artigo os autores focam a classificação dos segmentos obtidos através do desenvolvimento de duas ferramentas digitais que possibilitam o treino de algoritmos de classificação supervisionada. Estas ferramentas interagem com modelos BIM para a criação expedita de um elevado número de nuvens de pontos artificiais já categorizadas, possibilitando a criação de uma base de dados onde treinar o software de aprendizagem computacional.

Além desta secção introdutória, a estrutura do artigo é a seguinte: Na Secção 2 é descrita a metodologia aplicada no desenvolvimento deste artigo. A Secção 3 corresponde à revisão bibliográfica do tópico "Scan-to-BIM". A Secção 4 resume a metodologia proposta para a automatização do processo "Scan-to-BIM", focando o segundo passo da mesma. Finalmente, na Secção 5 são retiradas as conclusões do artigo e apresentados os trabalhos futuros.

\section{Metodologia}

Este artigo foi desenvolvido conforme a metodologia presente na Figura 1.

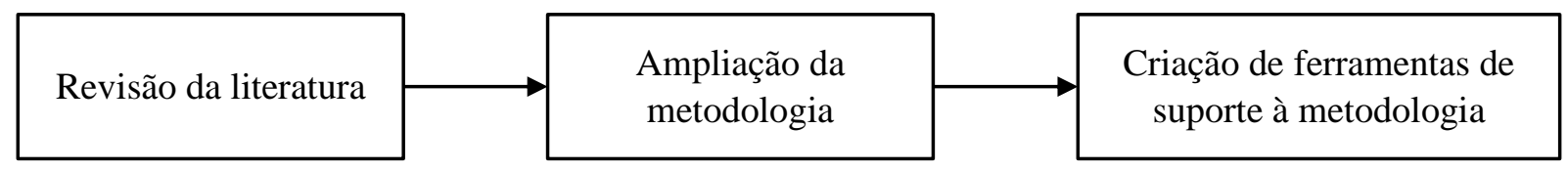

Figura 1: Metodologia seguida na criação deste artigo.

Inicialmente, uma revisão do estado-da-arte, focada no tópico de Scan-to-BIM, foi realizada com o objetivo de identificar atuais lacunas na literatura e fundamentar a necessidade da metodologia proposta. Com o conhecimento obtido nesta revisão, bem como a experiência prévia dos autores, procedeu-se à ampliação da metodologia previamente proposta para a automatização do processo "Scan-to-BIM" [1], focando a classificação dos segmentos obtidos. Finalmente, com o objetivo de facilitar a aplicação da metodologia proposta, foram criadas duas ferramentas de suporte à mesma. No que diz respeito ao software utilizado, o Autodesk Revit foi aplicado para a criação do modelo BIM, enquanto o Dynamo foi usado para a criação das ferramentas a incluir na metodologia. O Dynamo foi essencialmente utilizado pelo seu acesso à API (Application Programming Interface) do Revit, sendo que a linguagem de programação principal foi Python. Em relação ao hardware, o computador utilizado apresenta as seguintes especificações: CPU INTEL I7-6700K (4.00GHz); GPU MSI GeForce GTX 1050 Ti Gaming X 4G; RAM 32GB (2x16GB) DDR4-2400MHz CL15; Storage SSD 2.5' '500GB SATA 3.

\section{Estado da arte}

Nas últimas décadas, mais proeminentemente nos últimos anos com o surgimento do BIM, registou-se uma explosão de geração de informação no sector da Construção. Esta informação 
permite solucionar problemas críticos do sector, nomeadamente na segurança [12] e na gestão de infraestruturas [13]. Contudo, frequentemente, a informação é recolhida sem um propósito final, com bases de dados relevantes a permanecerem sem qualquer tipo de aplicação [14]. A aprendizagem computacional encontra-se numa posição ideal para usufruir destes dados, oferecendo aos computadores a capacidade de interpretar e aplicar esta informação, sem serem explicitamente programados para o fazer [15]. Neste sentido, ao rever as últimas três décadas de investigação relacionada com aprendizagem computacional no sector da Construção, é possível observar um crescimento exponencial do número de contributos nesta área (Figura 2 - string de pesquisa: "machine learning", com restrição às categorias de "Civil Engineering" e "Construction Building Technology"), na qual um dos atuais tópicos de maior interesse é a automatização do processo Scan-to-BIM.

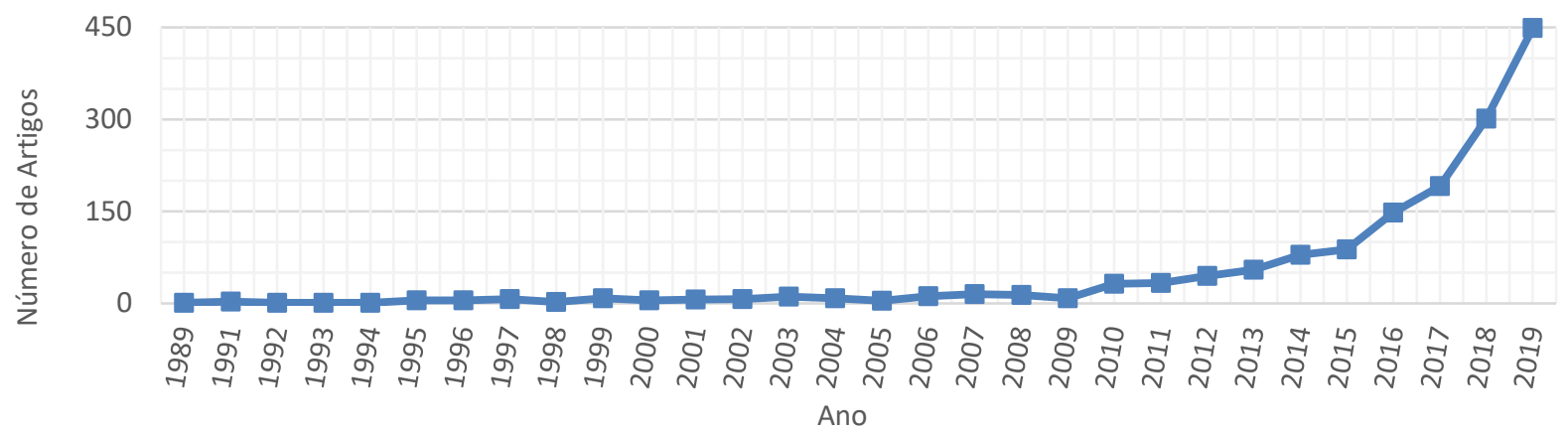

Figura 2: Número de artigos relacionados com aprendizagem computacional no sector da Construção de acordo com a Web of Science.

Como anteriormente referido, a literatura divide o processo de Scan-to-BIM em três passos principais. No primeiro, segmentação da nuvem de pontos, a literatura já conta com um número significativo de contributos, existindo uma clara divisão desta tarefa em duas partes: limpeza da nuvem; e segmentação. Na primeira parte, os trabalhos anteriores tipicamente começam por identificar os pontos associados a componentes estruturais (e.g. lajes, pilares, vigas), eliminando posteriormente os restantes pontos que, por exclusão, são considerados ruído [16]. Esta abordagem é aceite pela maioria dos autores como de fácil implementação e menor exigência em termos computacionais [16, 17], quando comparada com o processo inverso (ver [18]). Relativamente à segunda parte, a generalidade da investigação foca-se na geração de regiões através de aprendizagem computacional não supervisionada (treino com dados sem categorização prévia), tipicamente por algoritmos de densidade [16]. Exemplos desta abordagem podem ser vistos em [16, 19, 20], onde os autores aplicam variações do algoritmo K-Nearest Neighbours (KNN), em conjunto com distâncias Euclidianas e informação sobre a cor de cada ponto. Após esta geração de regiões inicial, algoritmos heurísticos dependentes de informação local e contextual, aperfeiçoam a forma destas regiões, ajustando as dimensões finais de cada região e criando os limites dos elementos construtivos [16]. Para mais informação nestes dois tipos de informação ver [7], com exemplos da sua aplicação em [21] e [22, 23], respetivamente para informação local e contextual. Alternativas a este processo podem ser vistas em [24] e [25], onde os autores utilizam sistemas de classificação restritiva e convenções gerais do setor da Construção (e.g. paralelismo entre lajes) para obter as dimensões finais.

No que diz respeito ao segundo e terceiro passo, classificação dos segmentos obtidos em elementos construtivos e posterior modelação BIM, a literatura encontra-se mais restringida. 
De facto, apesar de existirem alguns estudos com foco na classificação e modelação 3D [7, 8, 16, 26, 27], estes estudos restringem-se a estas duas tarefas, faltando o enriquecimento semântico e estrutural dos elementos. Desta forma, a generalidade da literatura atual apresenta apenas a uma modelação CAD 3D e não BIM. Adicionalmente, as tentativas de reconhecimento de elementos construtivos em nuvens de pontos restringem-se, tipicamente, a processos heurísticos, ontológicos, contextuais ou baseados em conhecimento prévio, que apresentam uma difícil escalabilidade e adaptação a casos particulares [8]. Exemplos destes tipos de abordagem podem ser vistos em [17, 24, 26-29]. Contrariamente, embora mais escassos, processos baseados em algoritmos de aprendizagem computacional supervisionada (treino com dados categorizados), apresentam uma maior adaptação. Estudos com foco nesta abordagem podem ser vistos em [30] e [31], onde os autores aplicam vários algoritmos de classificação supervisionados para a identificação de um número restrito de elementos construtivos, obtendo precisões entre os 60 e 90\%. Em [32], os autores apresentam algoritmos para a identificação de diferentes tipos de portas com resultados positivos, contudo, o processo de modelação não foi tentado. Similarmente, Adán et al. [33] atingiu taxas médias de $80 \%$ para a classificação de elementos "secundários" (e.g. alarmes de fogo, extintores), contudo, novamente, a sua modelação não foi tentada. Finalmente, em [16], os autores propõem um abordagem por empilhamento, utilizando as classificações de vários algoritmos para melhorar a classificação final dos elementos construtivos.

Concluindo, os estudos anteriormente citados apresentam resultados satisfatórios no reconhecimento e dimensionamento dos elementos estruturais principais (e.g. lajes e paredes), contudo, outros componentes (e.g. janelas, portas, colunas) são geralmente ignorados ou obtidos com taxas de reconhecimento demasiado baixas para justificar a utilização do software [31-33]. Adicionalmente, estes algoritmos são criados com base em regras tradicionais de engenharia e arquitetura (e.g. paralelismo entre lajes e paredes), o que resulta num viés desfavorável e uma dificultada adaptação a novos casos. Finalmente, a modelação automática dos elementos reconhecidos é raramente realizada, sendo que quando feita restringe-se a uma modelação CAD 3D e não BIM, faltando o enriquecimento semântico, contextual e estrutural dos elementos modelados.

\section{Metodologia para a criação artificial de nuvens de pontos}

A Figura 3 apresenta uma simplificação da metodologia previamente criada para a automatização do processo Scan-to-BIM [1], com foco no segundo passo da mesma: classificação dos segmentos obtidos em elementos construtivos. Como é visível nesta figura, a metodologia inicia-se pela aquisição da informação geométrica e não-geométrica de um edifício. A informação geométrica é obtida por laser scanning, resultando numa nuvem de pontos cujas dimensões serão utilizadas na modelação automática dos elementos construtivos em BIM. O segundo conjunto de informação pode ser proveniente de várias metodologias de aquisição de informação in-situ [34], tendo como objetivo o enriquecimento dos elementos construtivos automaticamente modelados. De seguida, é realizada a segmentação automática da nuvem de pontos, utilizando para isso algoritmos de aprendizagem computacional não supervisionados. Obtidos os segmentos, estes são classificados no segundo passo da metodologia em elementos construtivos ou ruído. No terceiro e último passo da metodologia, a 
informação não-geométrica anteriormente obtida é atribuída aos elementos construtivos identificados, que são automaticamente modelados em BIM.

Focando o segundo passo, para possibilitar a classificação dos segmentos obtidos em elementos construtivos ou ruído, é necessário treinar um classificador. Para isso os seguintes pontos foram delineados:

1. classificação manual dos segmentos anteriormente obtidos;

2. divisão dos segmentos em conjuntos de treino e teste;

3. extração de features do grupo de treino, seguida da sua seleção conforme a respetiva relevância para a classificação dos segmentos;

4. treino de múltiplos algoritmos de classificação (e.g. Decision Tree (DT); KNN; Logistic Regression (LR); Multilayer Perceptron (MLP); Multiclass Support Vector Machines (SVM) com diferentes kernels (linear (LSVM), polinomial (PSVM), rbf (RSVM), e sigmoid (SSVM)); Random Forest (RF); Extremely Randomized Trees (ExT); AdaBoost (AdB); Gradient Boosting (GrB)), incluindo hyperparameter tunning;

5. e, por fim, seleção do melhor classificador conforme a sua precisão e desempenho.

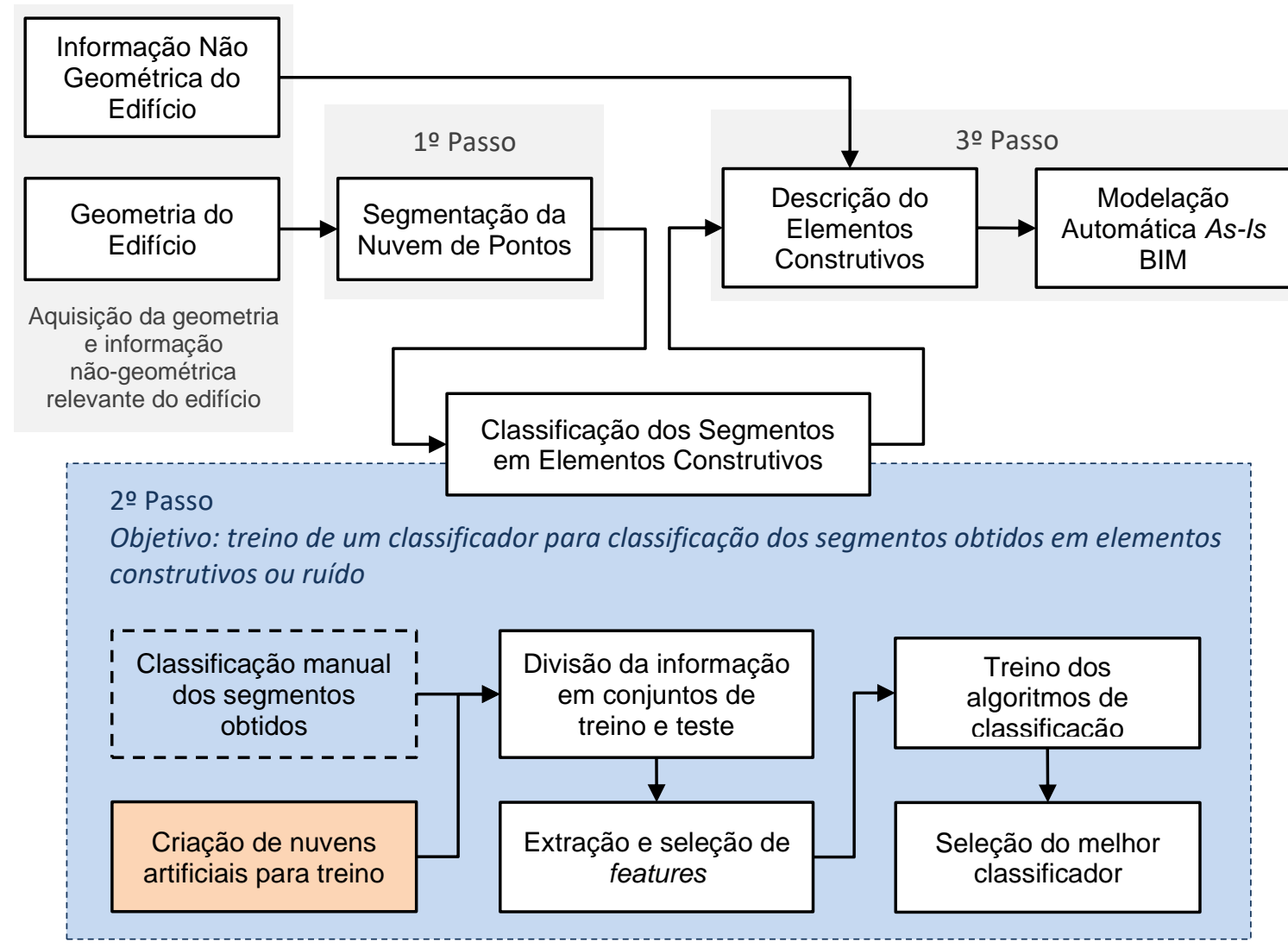

Figura 3: Metodologia para automação do processo Scan-to-BIM, com foco na classificação dos segmentos em elementos construtivos.

Contudo, a classificação manual de cada um dos segmentos em elementos construtivos ou ruído (ponto 1) é um processo demorado e exaustivo. Adicionalmente, para o treino dos algoritmos de classificação supervisionados é necessário um elevado número de segmentos, o que implica 
o levantamento laser scanner de múltiplos edifícios com diferentes níveis de complexidade e condições de observação. Neste sentido, com o intuito de ultrapassar este obstáculo, foram criadas duas ferramentas para a criação expedita de um elevado número de nuvens de pontos artificiais, já segmentadas e classificadas. Ambas as ferramentas apresentam funções essenciais para a automatização deste processo, nomeadamente: o posicionamento automático das estações laser scanner no modelo BIM; e a simulação do seu funcionamento, obtendo assim nuvens de pontos do modelo BIM. O funcionamento destas ferramentas é apresentado nas secções seguinte.

\subsection{Ferramenta para o posicionamento automático de estações laser scanner}

Como referido anteriormente, a primeira ferramenta tem como objetivo fazer um posicionamento automático das estações laser scanner dentro do modelo BIM. Desta forma, para criar uma nuvem de pontos artificial é apenas necessário abrir um modelo BIM e correr o programa, dispensando um planeamento prévio das estações. Este posicionamento é realizado com o intuito de obter a totalidade da geometria interna do edifício, com o objetivo adicional de fazê-lo de forma eficiente. Para isso, foi criada uma função custo (Equação 1) para avaliação do posicionamento do laser scanner. O conjunto de posições escolhido é o que simultaneamente adquire na totalidade a geometria interior do edifício e minimiza a função custo. Esta função tem por base testes de laboratório anteriormente realizados [35], apresentado como variáveis a qualidade (PCqlty) e resolução do scan (PCres), a resolução das fotografias (IMGres) (caso sejam tiradas), e o tamanho da equipa a fazer o levantamento (TEq). A primeira parcela desta equação é obtida pela Tabela 1 e corresponde à duração do scan (Duração). As durações do scan vêm tipicamente indicadas na documentação do laser scanner usado, habitualmente em função da qualidade e resolução escolhidas. Os valores presentes na Tabela 1 dizem respeito ao laser scanner ScanStation P20 da Leica.

$$
\begin{aligned}
\text { Custo }= & \text { Duração }(\text { PCqlty; PCres }) \\
& +843.513 / \text { PCres }^{1.978} \\
& +0.676 \times 10^{-5} \times \text { IMGres }^{2}+0.136 \times 10^{-2} \times \text { IMGres } \\
& -0.735 \times \ln (\text { TEq })+2.835
\end{aligned}
$$

Tabela 1: Duração do scan (minutos)

\begin{tabular}{lccccccc}
\hline \multirow{2}{*}{ Qualidade } & \multicolumn{7}{c}{ Resolução $(\mathbf{m m} @ \mathbf{1 0 m})$} \\
\cline { 2 - 8 } & $\mathbf{5 0 . 0}$ & $\mathbf{2 5 . 0}$ & $\mathbf{1 2 . 5}$ & $\mathbf{6 . 3}$ & $\mathbf{3 . 1}$ & $\mathbf{1 . 6}$ & $\mathbf{0 . 8}$ \\
\hline 1 & 0.333 & 0.550 & 0.967 & 1.817 & 3.500 & 13.550 & 54.117 \\
\hline 2 & 0.333 & 0.550 & 1.733 & 3.417 & 6.783 & 27.067 & 108.217 \\
\hline 3 & 0.467 & 0.883 & 3.400 & 6.767 & 13.500 & 54.117 & - \\
\hline 4 & - & 1.717 & 6.767 & 13.500 & 26.983 & - & - \\
\hline
\end{tabular}

Relativamente ao funcionamento da ferramenta em si, este consiste num processo heurístico e iterativo. A ferramenta começa por criar um conjunto igualmente espaçado de possíveis posições para o laser scanner. De seguida, estas posições são analisadas para que não exista nenhuma incompatibilidade entre o as dimensões do laser scanner e a geometria do edifício. Caso exista (e.g. localização demasiado estreita), posições incompatíveis são movidas para a a área compatível mais próxima. Terminado este processo, para cada uma das posições são 
criados campos de visão que simulam a área abrangida pelo laser scanner. Estes campos de visão são representados por esferas, cujo raio é obtido (em metros) pela Equação 2. Caso a esfera intersete a geometria do edifício, o campo de visão molda-se a esta mesma, igualando o comportamento do laser scanner. Posteriormente, uma análise combinatória destes campos é realizada, sendo a combinação escolhida a que simultaneamente cobre a geometria interna do edifício e minimiza a função custo. Por fim, novas posições são criadas em redor das posições escolhidas, repetindo o processo até novas soluções oferecerem ganhos insignificativo.

$$
\text { Raio }=\text { resolução_pretendida } \times 10 / \text { resolução_testada } a_{i}
$$

Nas Figuras 4 e 5 é possível observar um modelo BIM criado para teste desta ferramenta, bem como alguns conjuntos de posições criados e testados por esta.

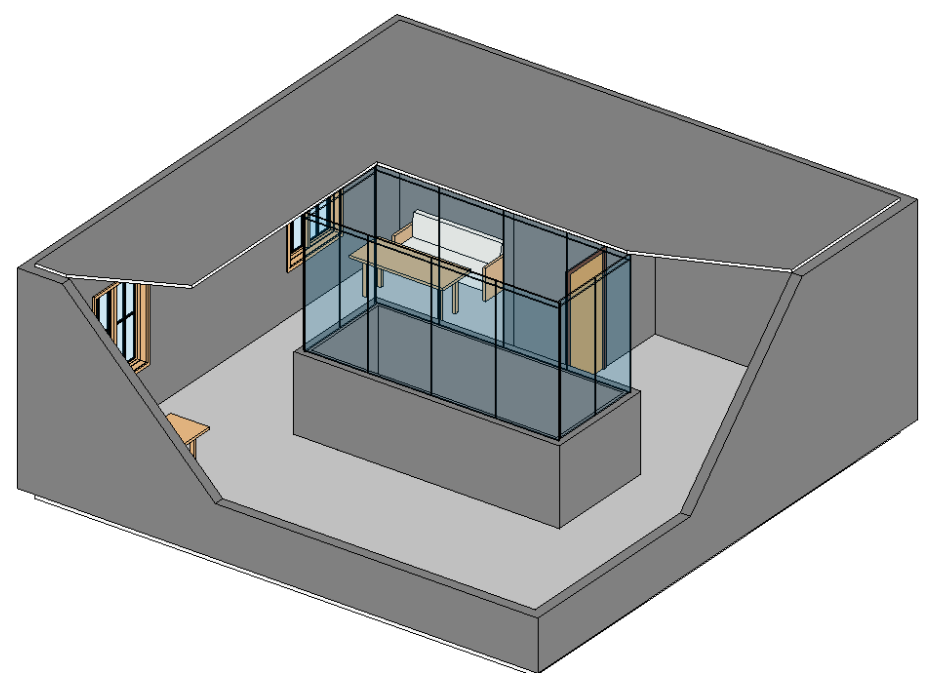

Figura 4: Modelo BIM no qual as ferramentas foram aplicadas.
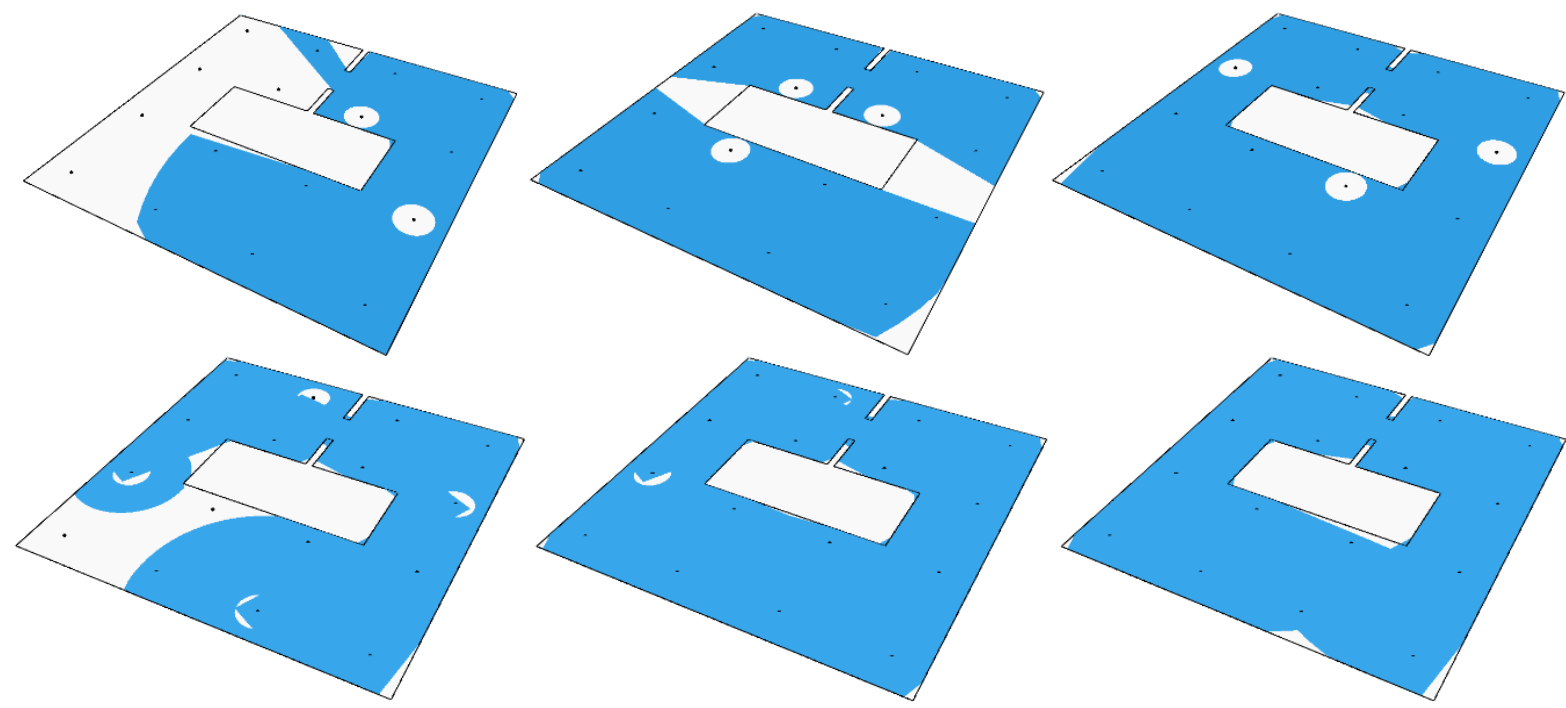

Figura 5: Possíveis conjuntos de posições criados e testados pela ferramenta para posicionamento automático de estações laser scanner. Campos de visão representados a azul. 


\subsection{Ferramenta para simulação do funcionamento de uma estação laser scanner}

Terminada a escolha da localização das estações laser scanner, bem como das suas respetivas resoluções, esta informação é utilizada pela segunda ferramenta para simular o seu funcionamento. $\mathrm{O}$ processo inicia-se pela criação de $n$ pontos a 1.6 metros de altura e coordenadas $\mathrm{X}$ e Y iguais às $n$ localizações escolhidas. Estes pontos representam a origem dos feixes do laser scanner a serem simulados. $\mathrm{O}$ valor default de 1.6 metros pode ser alterado pelo utilizador. De seguida, o primeiro feixe é criado paralelamente ao eixo $\mathrm{Y}$, tendo como comprimento o raio previamente calculado para a respetiva estação. Criado este feixe inicial, este sofre uma rotação horizontal centrada na sua origem (Figure 6A) e cujo ângulo é obtido (em graus) pela Equação 3. Esta operação é repetida até o feixe terminar uma rotação de $360^{\circ}$. Posteriormente, o mesmo ângulo é utilizado para fazer uma rotação vertical dos feixes obtidos (Figura 6B). Esta última rotação não cobre a totalidade dos $360^{\circ}$, percorrendo apenas o intervalo $[-45,225]$. Criados todos os feixes, sempre que estes intersetem a geometria presente no modelo BIM, a primeira interseção do feixe é registada como um ponto da nuvem. 0 ponto obtido é guardado numa lista relativa ao elemento construtivo intersetado, garantindo assim a classificação dos segmentos da nuvem de pontos obtida.

$$
\alpha=\tan ^{-1}(\text { resolução_escolhida } / 10)
$$

Nas Figuras 7 e 8 é possível observar o resultado da aplicação desta ferramenta a apenas uma posição do modelo BIM, originando uma nuvem de pontos artificial já categorizada.

$$
\text { A. }
$$

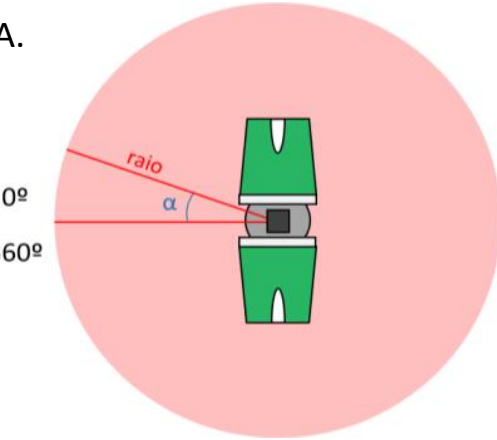

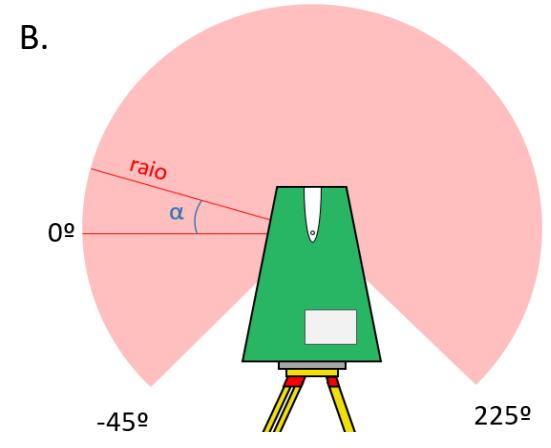

Figura 6: Rotação do feixe inicial. Vista de topo (A) e lateral (B).

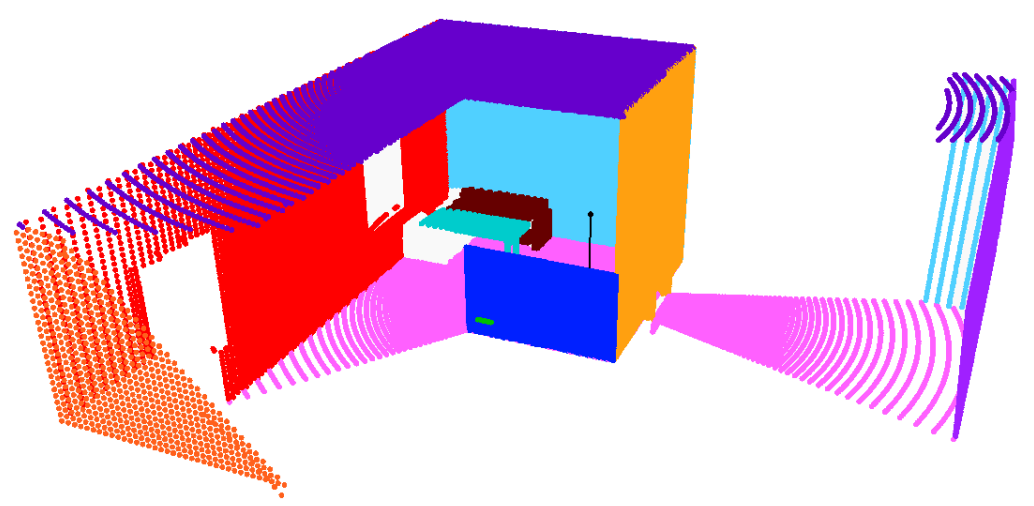

Figura 7: Nuvem de pontos categorizada originada pela ferramenta para simulação do funcionamento de uma estação laser scanner. 


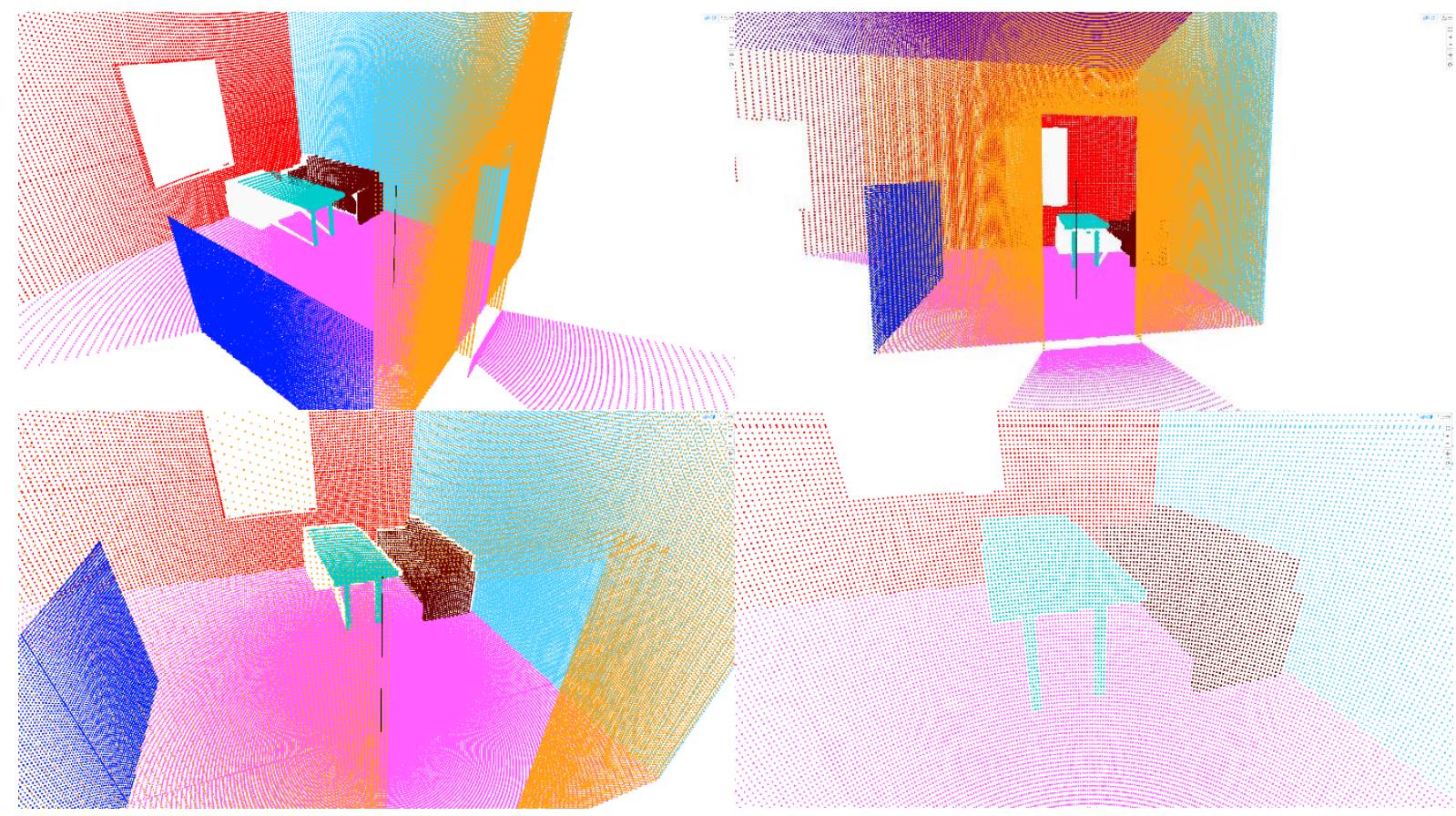

Figura 8: Detalhe da nuvem de pontos categorizada originada pela ferramenta para simulação do funcionamento de uma estação laser scanner.

\section{Conclusões e trabalhos futuros}

O presente artigo expôs o funcionamento de duas ferramentas BIM para a criação expedita de nuvens de pontos de elementos construtivos categorizados. As ferramentas apresentadas foram desenvolvidas no contexto de uma metodologia cujo objetivo passa pela modelação automática BIM de edifícios existentes. Como visível nas Figuras 4, 5, 7 e 8, estas ferramentas encontramse a funcionar corretamente, justificando a aplicação das mesmas em casos de estudo para validação. Em termos de trabalhos futuros, os autores utilizarão estas ferramentas para a criação expedita de uma base de dados de nuvens de pontos de elementos construtivos. Alguns dos elementos a abranger inicialmente serão: pavimentos; tetos; paredes; pilares expostos; vigas expostas; portas; e janelas. Todos estes elementos serão capturados tendo em conta um leque diversificado de ângulos e níveis de oclusão. Terminada a criação da base de dados, as nuvens de pontos serão utilizadas no treino e teste de algoritmos de aprendizagem computacional supervisionada para a identificação de elementos construtivos em nuvens de pontos.

\section{Reconhecimentos}

Este trabalho foi financiado por: Financiamento Base - UIDB/04708/2020 e Financiamento programático - UIDP/04708/2020 da Unidade de Investigação CONSTRUCT - Instituto de I\&D em Estruturas e Construções - financiada por fundos nacionais através da FCT/MCTES (PIDDAC). Bem como pelo financiamento da FCT através da Bolsa de Doutoramento SFRH/BD/129652/2017. 


\section{Referências}

[1] L. Sanhudo, J. P. Martins, and N. M. Ramos, "An Initial Approach to Automatic Building Information Modelling," 3rd Doctoral Congress in Engineering, 2019.

[2] FIEC, "Statistical Report - The Construction Activity in EUROPE," European Construction Industry Federation, 2019.

[3] A. Rocha et al., "A case study to improve the winter thermal comfort of an existing bus station," Journal of Building Engineering, p. 101123, 2019.

[4] L. Sanhudo et al., "Building information modeling for energy retrofitting-A review," Renewable and Sustainable Energy Reviews, vol. 89, pp. 249-260, 2018.

[5] L. Sanhudo et al., "A framework for in-situ geometric data acquisition using laser scanning for BIM modelling," Journal of Building Engineering, vol. 28, p. 101073, 2020.

[6] H. Macher, T. Landes, and P. Grussenmeyer, "From Point Clouds to Building Information Models: 3D Semi-Automatic Reconstruction of Indoors of Existing Buildings," Applied Sciences, vol. 7, no. 10, p. 1030, 2017.

[7] P. Tang, D. Huber, B. Akinci, R. Lipman, and A. Lytle, "Automatic reconstruction of asbuilt building information models from laser-scanned point clouds: A review of related techniques," Automation in construction, vol. 19, no. 7, pp. 829-843, 2010.

[8] N. Hichri, C. Stefani, L. De Luca, P. Veron, and G. Hamon, "From point cloud to BIM: a survey of existing approaches," in XXIV International CIPA Symposium, 2012, p. na: Proceedings of the XXIV International CIPA Symposium.

[9] D. Huber, B. Akinci, A. A. Oliver, E. Anil, B. E. Okorn, and X. Xiong, "Methods for automatically modeling and representing as-built building information models," in Proceedings of the NSF CMMI Research Innovation Conference, 2011.

[10]R. J. Scherer and P. Katranuschkov, "BIMification: How to create and use BIM for retrofitting," Advanced Engineering Informatics, vol. 38, pp. 54-66, 2018.

[11]L. Barazzetti, F. Banfi, R. Brumana, and M. Previtali, "Creation of parametric BIM objects from point clouds using NURBS," The Photogrammetric Record, vol. 30, no. 152, pp. 339$362,2015$.

[12]V. Bansal, "Application of geographic information systems in construction safety planning," International Journal of Project Management, vol. 29, no. 1, pp. 66-77, 2011.

[13] N. R. Council and N. Kassabian, Transportation Data and Information Systems: Current Applications and Needs, 1990. Transportation Research Board, 1990.

[14] Y. Reich, "Machine learning techniques for civil engineering problems," Computer-Aided Civil and Infrastructure Engineering, vol. 12, no. 4, pp. 295-310, 1997.

[15] S. Lee, J. Ha, M. Zokhirova, H. Moon, and J. Lee, "Background Information of Deep Learning for Structural Engineering," Archives of Computational Methods in Engineering, vol. 25, no. 1, pp. 121-129, 2018.

[16]X. Xiong, A. Adan, B. Akinci, and D. Huber, "Automatic creation of semantically rich 3D building models from laser scanner data," Automation in Construction, vol. 31, pp. 325337, 2013.

[17]E. Turner and A. Zakhor, "Automatic indoor 3D surface reconstruction with segmented building and object elements," in 3D Vision (3DV), 2015 International Conference on, 2015, pp. 362-370: IEEE.

[18] K. Ishida, N. Kano, and K. Kimoto, "Shape recognition with point clouds in rebars," in ISARC. Proceedings of the International Symposium on Automation and Robotics in 
Construction, 2012, vol. 29, p. 1: Vilnius Gediminas Technical University, Department of Construction Economics ....

[19]X. Ning, X. Zhang, Y. Wang, and M. Jaeger, "Segmentation of architecture shape information from 3D point cloud," in Proceedings of the 8th International Conference on Virtual Reality Continuum and its Applications in Industry, 2009, pp. 127-132: ACM.

[20]Q. Zhan, Y. Liang, and Y. Xiao, "Color-based segmentation of point clouds," Laser scanning, vol. 38, no. 3, pp. 155-161, 2009.

[21]B. K. Horn and K. Ikeuchi, "The mechanical manipulation of randomly oriented parts," Scientific American, vol. 251, no. 2, pp. 100-113, 1984.

[22] H. Cantzler, "Improving architectural 3D reconstruction by constrained modelling," 2003.

[23] A. Nüchter and J. Hertzberg, "Towards semantic maps for mobile robots," Robotics and Autonomous Systems, vol. 56, no. 11, pp. 915-926, 2008.

[24] C. Wang, Y. K. Cho, and C. Kim, "Automatic BIM component extraction from point clouds of existing buildings for sustainability applications," Automation in Construction, vol. 56, pp. 1-13, 2015/08/01/ 2015.

[25]E. Valero, A. Adán, and C. Cerrada, "Automatic method for building indoor boundary models from dense point clouds collected by laser scanners," Sensors, vol. 12, no. 12, pp. 16099-16115, 2012.

[26] I. Anagnostopoulos, M. Belsky, and I. Brilakis, "Object Boundaries and Room Detection in As-Is BIM Models from Point Cloud Data," in Proceedings of the 16th International Conference on Computing in Civil and Building Engineering, Osaka, Japan, 2016, pp. 68.

[27]C. Thomson and J. Boehm, "Automatic geometry generation from point clouds for BIM," Remote Sensing, vol. 7, no. 9, pp. 11753-11775, 2015.

[28]X. Xiong and D. Huber, "Using Context to Create Semantic 3D Models of Indoor Environments," in BMVC, 2010, pp. 1-11.

[29] S. Pu and G. Vosselman, "Extracting windows from terrestrial laser scanning," Intl Archives of Photogrammetry, Remote Sensing and Spatial Information Sciences, vol. 36, pp. 12-14, 2007.

[30] M. Bassier, B. Van Genechten, and M. Vergauwen, "Classification of sensor independent point cloud data of building objects using random forests," Journal of Building Engineering, vol. 21, pp. 468-477, 2019/01/01/ 2019.

[31]J. Jung, C. Stachniss, S. Ju, and J. Heo, "Automated 3D volumetric reconstruction of multiple-room building interiors for as-built BIM," Advanced Engineering Informatics, vol. 38, pp. 811-825, 2018.

[32]B. Quintana, S. Prieto, A. Adán, and F. Bosché, "Door detection in 3D coloured point clouds of indoor environments," Automation in Construction, vol. 85, pp. 146-166, 2018.

[33] A. Adán, B. Quintana, S. A. Prieto, and F. Bosché, "Scan-to-BIM for 'secondary' building components," Advanced Engineering Informatics, vol. 37, pp. 119-138, 2018/08/01/ 2018.

[34]L. Sanhudo et al., "Building information modeling for energy retrofitting - A review," Renewable and Sustainable Energy Reviews, vol. 89, pp. 249-260, 2018/06/01/ 2018.

[35]L. Sanhudo et al., "A framework for in-situ geometric data acquisition using laser scanning for BIM modelling," Journal of Building Engineering, vol. 28, p. 101073, 2020/03/01/ 2020 . 